\title{
Semi-automated non-target processing in GC $\times$ GC-MS metabolomics analysis: applicability for biomedical studies
}

\author{
Maud M. Koek • Frans M. van der Kloet • \\ Robert Kleemann - Teake Kooistra • \\ Elwin R. Verheij · Thomas Hankemeier
}

Received: 14 February 2010/ Accepted: 25 May 2010/Published online: 15 July 2010

(C) The Author(s) 2010. This article is published with open access at Springerlink.com

\begin{abstract}
Due to the complexity of typical metabolomics samples and the many steps required to obtain quantitative data in GC $\times$ GC-MS consisting of deconvolution, peak picking, peak merging, and integration, the unbiased nontarget quantification of GC $\times$ GC-MS data still poses a major challenge in metabolomics analysis. The feasibility of using commercially available software for non-target processing of $\mathrm{GC} \times \mathrm{GC}-\mathrm{MS}$ data was assessed. For this purpose a set of mouse liver samples (24 study samples and five quality control (QC) samples prepared from the study samples) were measured with GC $\times$ GC-MS and GC-MS to study the development and progression of insulin resistance, a primary characteristic of diabetes type 2 . A total of 170 and 691 peaks were quantified in, respectively, the GC-MS and GC $\times$ GC-MS data for all study and QC samples. The quantitative results for the $\mathrm{QC}$ samples were compared to assess the quality of semi-automated
\end{abstract}

Electronic supplementary material The online version of this article (doi:10.1007/s11306-010-0219-6) contains supplementary material, which is available to authorized users.

M. M. Koek $(\square)$ · E. R. Verheij

Analytical Research Department, TNO Quality of Life,

Utrechtseweg 48, 3704 HE Zeist, The Netherlands

e-mail: maud.koek@tno.nl

R. Kleemann · T. Kooistra

Department of Vascular and Metabolic Disease, TNO Quality

of Life, Zernikedreef 9, 2333 CK Leiden, The Netherlands

F. M. van der Kloet · T. Hankemeier

LACDR Analytical Biosciences, Leiden University,

Einsteinweg 55, 2333 CC Leiden, The Netherlands

T. Hankemeier

Netherlands Metabolomics Centre, Einsteinweg 55,

2333 CC Leiden, The Netherlands
GC $\times$ GC-MS processing compared to targeted GC-MS processing which involved time-consuming manual correction of all wrongly integrated metabolites and was considered as golden standard. The relative standard deviations (RSDs) obtained with $\mathrm{GC} \times \mathrm{GC}-\mathrm{MS}$ were somewhat higher than with GC-MS, due to less accurate processing. Still, the biological information in the study samples was preserved and the added value of $\mathrm{GC} \times \mathrm{GC}-$ MS was demonstrated; many additional candidate biomarkers were found with $\mathrm{GC} \times \mathrm{GC}-\mathrm{MS}$ compared to GC-MS.

Keywords Metabolomics - Comprehensive two-dimensional gas chromatography mass spectrometry . $\mathrm{GC} \times \mathrm{GC}-\mathrm{MS} \cdot$ Automated data processing $\cdot$ Diabetes . Insulin resistance

\section{Introduction}

Metabolomics research involves the comprehensive nontarget analysis of all, or at least as many as possible, metabolites in cells, tissue or body fluids. The complexity of the metabolome makes this a challenging task for analytical chemists. For example, samples of the simplest microorganisms already contain, by estimation, several hundreds of different metabolites. At present, the main analytical techniques used for the analysis of the metabolome are nuclear magnetic resonance spectroscopy (NMR) and hyphenated techniques, such as gas chromatography (GC) and liquid chromatography (LC) coupled to mass spectrometry (MS).

Gas chromatography coupled to mass spectrometry (GC-MS) is a highly suitable technique for metabolomics analysis due to the high separation power, reproducible retention times and sensitive selective mass detection. 
In previous papers a one-dimensional GC-MS method (Koek et al. 2006) and a comprehensive two-dimensional gas chromatography mass spectrometry method $(\mathrm{GC} \times$ GC-MS; Koek et al. 2008) suitable for the analysis of a broad range of small polar metabolites were described using a derivatization with an oximation reagent followed by silylation. Several other GC-MS (Fiehn et al. 2000; Jonsson et al. 2004; Roessner et al. 2000; Strelkov et al. 2004; Villas-Boas et al. 2005) and GC $\times$ GC-MS (O'Hagan et al. 2007; Pierce et al. 2006b; Shellie et al. 2005) based methods for metabolomics have been reported.

The principle of GC $\times$ GC-MS is based on the coupling of two analytical columns with different selectivities coupled through a modulator. The so-called dual-stage cryogenic modulator equipped with four jets (two liquidnitrogen cooled and two hot-gas jets) allows for the consecutive trapping, cryogenic focussing and release of small fractions from the first column effluent in narrow bands onto the second column. In this comprehensive setup, the entire sample is separated on both columns and no information of the first separation is lost during the second one. The resulting GC $\times$ GC-MS chromatogram consists of a large series of consecutive second dimension $\left({ }^{2} \mathrm{D}\right)$ separations. To maintain the separation of the first column, each peak eluting from the first dimension should be sampled, i.e. modulated, minimally three to four times (Murphy et al. 1998).

GC $\times$ GC-MS offers several advantages over GC-MS, i.e. higher chromatographic separation power, a broader dynamic range and lower detection limits, and should be the preferred technique for metabolomics analysis. However, quantification of metabolomics samples using $\mathrm{GC} \times \mathrm{GC}-\mathrm{MS}$ is still a major challenge. To get from raw total-ion chromatographic data to a list of sample components with their corresponding peak areas and mass spectra, many steps are required, including peak finding, deconvolution, integration and combining of the peaks from different modulations originating from one compound. The performances of all the steps are influencing the final data quality and, consequently, the reliability of the biological information extracted from the data. In addition, all metabolites are of interest and need to be quantified. Several approaches have been published to process $\mathrm{GC} \times \mathrm{GC}-$ MS data after analysis to find metabolites that distinguish between samples (Mohler et al. 2008; Pierce et al. 2006a; Shellie et al. 2001; Sinha et al. 2004), but only few papers on the quantification of all (or at least as many as possible peaks) peaks have been published.

Hoggard and Synovec (2008) described a method for applying PARAFAC to GC $\times$ GC-TOF-MS data in an automated fashion that required no assumptions about analyte identities. They proposed that the method was applicable as post processing step providing deconvolution and quantification of all analytes in a sample.
However, their method was very time-consuming, i.e. one chromatogram had to be divided in numerous subsections, and complete analysis required, by their estimation, tens of hours. Oh et al. (2008) developed a peak sorting method (MSsort) for GC $\times$ GC-MS data in Matlab. Raw data files were first processed using the ChromaTOF software (LECO, St. Joseph, MI, USA) to provide peak tables. Subsequently, MSsort was used to sort and combine peaks by utilizing first- $\left({ }^{1} \mathrm{r}_{\mathrm{T}}\right)$ and second-dimension $\left({ }^{2} \mathrm{r}_{\mathrm{T}}\right)$ retention times and the mass spectrum. However, no quantitative data were presented in neither of the described papers. To our knowledge the only attempt for non-target quantification of metabolites in a real life metabolomics study was published by Li et al. (2009). They quantified 692 peaks in 79 human-plasma samples to identify possible biomarkers for type-2 diabetes mellitus. Quantification was performed by exporting $\mathrm{m} / \mathrm{z} 73$ from the GC $\times$ GC-MS chromatograms and alignment, peak merging and quantification was performed using their in-house developed software (GC × GC Workstation; Qiu et al. 2007). The repeatability of the quantification was tested using pooled plasma samples. The mean relative standard deviations (RSDs) in five consecutive injections of one plasma sample and five consecutive injections of five different plasma samples were 14 and $20 \%$, respectively. It is not fully clear how many peaks were included in the mean RSD (only peaks that were quantified in all samples were included). Besides, the use of a single mass trace $(\mathrm{m} / \mathrm{z})$ instead of the deconvoluted spectrum of a peak for quantification can result in errors in quantification of coeluting peaks and the assignment of the identity of a peak.

In this paper, the possibilities and limitations of the software with regard to non-target semi-automated processing of GC $\times$ GC-MS data were evaluated. This was done by measuring and processing a set of mouse-liver samples that were part of a larger study investigating the development of insulin resistance/type-2 diabetes mellitus (DM2) (Kleemann et al. 2010). DM2 is a multifactorial complex disease associated with metabolic deregulations. Despite major efforts, the pathophysiological mechanisms underlying the beginning and progression of the disease are still incompletely understood. Identification of changes in hepatic metabolite profiles can help to identify dysregulated metabolic pathways in DM2 and thus in the selection of (new) therapeutic regimens. Mice with a humanized lipoprotein metabolism, APOE*3Leiden transgenic (E3L) mice (Zadelaar et al. 2007) were used and mice were fed a high-fat diet known to induce insulin resistance/DM2. Livers were collected at different time points during 12 weeks of high-fat-diet feeding. The samples were measured with $\mathrm{GC} \times \mathrm{GC}-\mathrm{MS}$ and with $\mathrm{GC}-\mathrm{MS}$ to be able to compare the results from both methods. Results of semi-automated $\mathrm{GC} \times \mathrm{GC}-\mathrm{MS}$ data processing were 
compared with a fully optimized, but labour-intensive, targeted GC-MS data processing method used in our lab, involving the inspection and, if required, manual correction of the integration of all quantified metabolites. In addition, time-resolved changes in metabolic profiles of the mouse livers were identified using principal-component analysis (PCA) and principal-component-discriminant analysis (PCDA).

\section{Experimental}

\subsection{Chemicals and materials}

Pyridine (Baker analyzed) was purchased from Mallinkrodt Baker (Deventer, The Netherlands) and pyridine hydrochloride (analytical grade) was purchased from SigmaAldrich (Zwijndrecht, The Netherlands). A solution of $56 \mathrm{mg} / \mathrm{ml}$ ethoxyamine hydrochloride ( $>99 \%$, Acros Organics, Geel, Belgium) in pyridine was used for oximation and $N$-methyl- $N$-trimethylsilyl trifluoroacetamide (MSTFA; Alltech, Breda, The Netherlands) was used for silylation.

\subsection{Standards}

Standards used as quality-control standards, leucine-d3, glutamic acid-d3, phenylalanine-d5, glucose-d7, alanine-d4 and cholic acid-d4, were purchased from Spectra Stable Isotopes (Columbia, USA). 4,4-Difluorobiphenyl, trifluoroantracene and dicyclohexyl phthalate were purchased from Sigma-Aldrich. Three internal standard (IS) mixtures were prepared; IS mix 1 containing leucine-d3 $(250 \mathrm{ng} / \mu \mathrm{l})$, glutamic acid-d3 (250 ng/ $\mu$ l), phenylalanine-d5 (250 ng/ $\mu \mathrm{l})$, glucose-d7 $(250 \mathrm{ng} / \mu \mathrm{l})$ in water, IS mix 2 containing alanine-d4 $(250 \mathrm{ng} / \mu \mathrm{l})$ and cholic acid-d4 $(250 \mathrm{ng} / \mu \mathrm{l})$ in pyridine, and IS mix 3 containing 4,4-difluorobiphenyl $(250 \mathrm{ng} / \mu \mathrm{l})$, trifluoroantracene $(250 \mathrm{ng} / \mu \mathrm{l})$ and dicyclohexyl phthalate $(250 \mathrm{ng} / \mu \mathrm{l})$ in pyridine.

\subsection{Mouse-liver samples}

Animal experiments were approved by the Institutional Animal Care and Use Committee of The Netherlands Organization for Applied Scientific Research (TNO) and were in compliance with European-Community specifications regarding the use of laboratory animals. Male ApoE*3Leiden transgenic (E3L) mice subjected to high fat diet feeding essentially as specified in Kleemann et al. (2010). Briefly, E3L mice displaying a humanized lipoprotein metabolism and lipid profile and sensitive to high fat diet treatment (Kleemann et al., 2007) were fed a highfat diet containing $24 \%$ beef tallow (HF diet; Hope Farms,
Woerden, The Netherlands) and were euthanized with $\mathrm{CO} / \mathrm{CO}_{2}$ after zero weeks $(n=8), 6$ weeks $(n=8)$ and 12 weeks $(n=8)$ of high-fat diet feeding.

Livers were collected at sacrifice and were snap-frozen immediately in liquid nitrogen, and stored at $-80^{\circ} \mathrm{C}$ until use (no longer than 10 months).

\subsection{Sample preparation}

The liver samples were freeze-dried overnight and homogenized. 10-mg aliquots of the liver samples were weighed and placed inside a 2-ml Eppendorf tube. After addition of $10 \mu \mathrm{l}$ of IS mix 1 and $500 \mu \mathrm{l}$ of methanol/water $4: 1 \mathrm{v} / \mathrm{v}$, all samples were sonificated for $30 \mathrm{~min}$ and subsequently centrifuged for $10 \mathrm{~min}$ at $14086 \times g(10000 \mathrm{rpm})$. The supernatants were transferred to autosampler vials and subsequently dried under nitrogen flow. Then $10 \mu \mathrm{l}$ IS mix 2 and $30 \mu$ l ethoxyamine hydrochloride solution were added and the samples were oximated for $90 \mathrm{~min}$ at $40^{\circ} \mathrm{C}$ on a tube roller mixer placed inside an oven. Subsequently, $10 \mu \mathrm{l}$ of IS mix 3 and $100 \mu \mathrm{l}$ of MSTFA were added and the samples were silylated for $50 \mathrm{~min}$ at $40^{\circ} \mathrm{C}$ on a tube roller mixer inside an oven. Finally, the samples were centrifuged for $20 \mathrm{~min}$ at $2081 \times g(3500 \mathrm{rpm})$ prior to injection.

\subsection{Quality-control (QC) sample}

A pooled sample of six different liver samples from different time points (two per time point) was used as QC sample. The samples were prepared according to the sample preparation described above; however, after extraction the supernatants of all samples were mixed and subsequently divided over ten separate autosampler vials. Furthermore, the amounts of liver sample and IS-mix were adjusted to obtain the same amount of biomass and internal standards in the QC samples compared to the study samples.

\subsection{GC-MS analysis}

The derivatized extracts were analyzed with an Agilent 6890 gas chromatograph coupled with an Agilent 5973 mass-selective detector (Agilent technologies, Santa Clara, CA, USA). 1- $\mu$ l aliquots of the extracts were injected into a DB5-MS capillary column $(30 \mathrm{~m} \times 250 \mu \mathrm{m}$ I.D., $0.25 \mu \mathrm{m}$ film thickness; J\&W Scientific, Folson, CA, USA) using PTV-injection (Gerstel CIS4 injector; Mülheim an der Ruhr, Germany) in the splitless mode. The temperature of the PTV was $70^{\circ} \mathrm{C}$ during injection and 0.6 min after injection the temperature was raised to $300^{\circ} \mathrm{C}$ at a rate of $2^{\circ} \mathrm{C} / \mathrm{s}$ and held at $300^{\circ} \mathrm{C}$ for $20 \mathrm{~min}$. The initial GC oven temperature was $70^{\circ} \mathrm{C}, 5 \mathrm{~min}$ after injection the GC-oven temperature was increased with $5^{\circ} \mathrm{C} / \mathrm{min}$ to $320^{\circ} \mathrm{C}$ and held 
for $5 \mathrm{~min}$ at $320^{\circ} \mathrm{C}$. Helium was used as a carrier gas, and pressure programmed such that the helium flow was kept constant at a flow rate of $1.7 \mathrm{ml}$ per min. Detection was achieved using MS detection in electron ionisation and full-scan monitoring mode $(\mathrm{m} / \mathrm{z} 15-800)$. The temperature of the ion source was set at $250^{\circ} \mathrm{C}$ and that of the quadrupole at $200^{\circ} \mathrm{C}$.

\subsection{GC $\times$ GC-MS analysis}

The derivatized samples were analyzed with an Agilent 6890 gas chromatograph fitted with a dual-stage, four-jet (two liquid-nitrogen cooled and two hot-gas jets) cryogenic modulator and a secondary oven (LECO) and coupled to a time-of-flight mass spectrometer (Pegasus III, LECO). The configuration of the first $\left({ }^{1} \mathrm{D}\right)$ and second dimension $\left({ }^{2} \mathrm{D}\right)$ column and the method parameters were optimized, as described in Koek et al. (2008).

A $30 \mathrm{~m} \times 0.25 \mathrm{~mm}$ I.D. $\times 0.25 \mu \mathrm{m}$ forte BPX-50 column (SGE, Milton Keynes, UK) was used as the ${ }^{1} \mathrm{D}$ column and a $2 \mathrm{~m} \times 0.32 \mathrm{~mm}$ I.D. $\times 0.25 \mu \mathrm{m}$ forte BPX5 column (SGE Europe) was used as the ${ }^{2} \mathrm{D}$ column.

1- $\mu \mathrm{l}$ aliquots of the derivatized extracts were injected using PTV-injection (Gerstel CIS4) in the splitless mode. The temperature of the PTV was $70^{\circ} \mathrm{C}$ during injection and 0.6 min after injection the temperature was raised to $300^{\circ} \mathrm{C}$ at a rate of $2^{\circ} \mathrm{C} / \mathrm{s}$ and held at $300^{\circ} \mathrm{C}$ for $20 \mathrm{~min}$. The initial GC-oven temperature was $70^{\circ} \mathrm{C}, 3 \mathrm{~min}$ after injection the temperature was raised to $300^{\circ} \mathrm{C}$ with a rate of $5^{\circ} \mathrm{C} / \mathrm{min}$ and held at $300^{\circ} \mathrm{C}$ for $10 \mathrm{~min}$. The temperature offset of the secondary oven and modulator compared to the GC oven were set at +30 and $+40^{\circ} \mathrm{C}$, respectively. The modulation time was $6 \mathrm{~s}$, with the hot-pulse time set at $1 \mathrm{~s}$. Helium was used as carrier gas and the analyses were carried out in constant-pressure mode at $300 \mathrm{kPa}$. The MS transfer line was set at $325^{\circ} \mathrm{C}$ and the ion-source temperature was $280^{\circ} \mathrm{C}$. The detector voltage was set at $-1600 \mathrm{~V}$ and the data acquisition rate was $75 \mathrm{~Hz}$.

\subsection{Data processing GC-MS}

The Chemstation software (Version E02.00.493, Agilent Technologies) was used for processing of the data. A target table was constructed using an in-house library containing the mass spectra and retention times of over 600 reference metabolites (authentic standards), over 100 annotated metabolites (spectral match with NIST library) and over 200 unknown metabolites commonly found in blood products. Furthermore, metabolites (known or unknown) specific for this study were added to the target table. A total of 175 targets were found in the QC samples (total of three) and quantified in all samples by reconstructing an ion chromatogram of a specific mass from the mass spectrum of the target. The quantification for all targets was manually checked by visual control and if necessary peak integration was corrected manually.

\subsection{Optimization of GC $\times$ GC-MS data processing}

ChromaTOF software V3.35 was used for data processing. During the optimization step the following parameters were varied separately in the processing method: first dimension peak width $\left({ }^{1} \mathrm{w}_{\mathrm{B}}\right)(30,60,90$ and $120 \mathrm{~s})$, second dimension peak width $\left({ }^{2} \mathrm{w}_{\mathrm{B}}\right)(0.1,0.15,0.2,0.3$ and $0.4 \mathrm{~s})$, smoothing factor (auto, $3,5,7$ ) and the match required to combine different ${ }^{2} \mathrm{D}$ peaks originating from one entry (400-800). The different processing methods were evaluated by investigating the deconvoluted mass spectra, the integration and the combining of the ${ }^{2} \mathrm{D}$ peaks of the IS. For all IS, except for cholic acid-d4, the naturally-occurring non-labelled form was also detected in the sample and partly coeluted with the labelled IS. These naturallyoccurring compounds (except for glucose that was present in extremely high concentration) were also evaluated to check the performance of the deconvolution. The IS and naturally-occurring metabolites were distributed over the entire chromatogram and eluted at ${ }^{1} \mathrm{r}_{\mathrm{T}}$ between 356 and $2846 \mathrm{~s}$ and ${ }^{2} \mathrm{r}_{\mathrm{T}}$ between 2.4 and $5 \mathrm{~s}$. The ${ }^{2} \mathrm{w}_{\mathrm{B}}$ and the match required to combine ${ }^{2} \mathrm{D}$ peaks were the primary parameters determining the quality of the deconvolution $\left({ }^{2} \mathrm{w}_{\mathrm{B}}\right)$ and the combining of the different ${ }^{2} \mathrm{D}$ peaks from one peak (both parameters). Unfortunately, it is not possible to set different ${ }^{2} \mathrm{~W}_{\mathrm{B}}$ in the software for different ${ }^{2} \mathrm{r}_{\mathrm{T}}$, because metabolites eluting at high ${ }^{2} \mathrm{r}_{\mathrm{T}}$, e.g. cholic acid-d4, were better quantified with broader peak widths than metabolites eluting at low ${ }^{2} \mathrm{r}_{\mathrm{T}}$. In our case study, the ${ }^{2} \mathrm{~W}_{\mathrm{B}}$ was best set somewhat narrower $(0.15 \mathrm{~s})$ than the actual peak width of the narrowest peaks of interest ( $0.2 \mathrm{~s}$ baseline).

\subsection{Data processing for $\mathrm{GC} \times \mathrm{GC}-\mathrm{MS}$}

A computer with the following specifications was used: Pentium [R] dual Intel processor CPU $3.4 \mathrm{GHz}, 3 \mathrm{~GB}$ RAM, hard disk: Serial ATA, 7200 RPM, 16 MB cache, RAID 24/7 (Seagate Barracuda ES, 3.0 GB/s, 500 GB). All samples were processed with ChromaTOF V3.35 software with the following settings. Baseline tracking: default; baseline offset: 1.0 ; peak width: $0.15 \mathrm{~s}$; segmented processing: peak find S/N 20, number of apexing masses 2; $\mathrm{GC} \times \mathrm{GC}$ parameters: match required to combine 500 , peak width $90 \mathrm{~s}$, mass threshold 0 . Quantification for every individual entry was performed on their unique mass in the mass spectrum determined by the ChromaTOF software. The peaks from the constructed calibration table were quantified in all QC and study samples. 


\subsection{Construction of calibration table for} $\mathrm{GC} \times \mathrm{GC}-\mathrm{MS}$

One of the QC samples from the middle of the sequence was processed with the method described above, except the peak find $\mathrm{S} / \mathrm{N}$ was set to 200 . As many artefact peaks as possible were removed. For example, all peaks related with solvents and reagents (eluting at low ${ }^{2} \mathrm{r}_{\mathrm{T}}$ ) and multiple entries from highly concentrated tailing metabolites (i.e. phosphate). All remaining entries were added to a calibration table. Targets from the 1D-GC-MS target table that were unambiguously identified in the $2 \mathrm{D}-\mathrm{GC} \times \mathrm{GC}-\mathrm{MS}$ data, i.e. the identity was confirmed by the injection of a authentic standard or the mass spectrum of the metabolite was considered unique, were renamed (total 107 targets) in the $2 \mathrm{D}$ calibration table. The maximum ${ }^{1} \mathrm{r}_{\mathrm{T}}$ deviation in the calibration table was set to $13 \mathrm{~s}$ for every entry. The retention time deviation was set to $0.1 \mathrm{~s}$, the minimum area threshold was 0 , the match threshold was 550 and the $\mathrm{S} / \mathrm{N}$ threshold was set to 5 .

\subsection{Post processing of GC $\times$ GC-MS}

The quantitative data for all 1025 targets in the calibration table were exported to Excel. Compounds that were not found in more than one QC sample were removed (825 entries left). Subsequently, entries with more than four blank values in all samples were removed from the data set (691 entries left excl. internal standards). Of course, a blank value can be obtained when the concentration of the metabolite is below the limit of detection. However, in many occasions blank values were obtained even when the peak of interest was present in the sample (further referred to as a missing value), due to a low spectrum match. A low match was mostly caused by mistakes in the deconvolution either in the sample itself or in the sample used for the construction of the calibration table. However, the use of a selective mass from the mass spectrum for every metabolite (as defined in the reference table of the selected QC sample), still allows the quantification of wrongly deconvoluted peaks, although the reliability is lower. To fill the remaining missing values in the data set (total of 169 blank values), the chromatograms were reprocessed with a match threshold in the calibration table of 200 rather than 500. In this way the missing values for peaks that were unassigned due to a low match factor could be filled from the newly processed data. Of course, only correct assignments of these missing peak areas (as manually controlled via correct mass spectrum and retention time) were filled from the newly processed data. Then, all remaining peaks with missing values in the $\mathrm{QC}$ and/or study samples were checked and corrected manually by assigning the right peak in the chromatogram to the compound in the calibration table. The integration of the peaks and the combining of ${ }^{2} \mathrm{D}$ peaks were not corrected as this was extremely time-consuming and therefore considered an unrealistic option.

\section{Results and discussion}

The present study was directed at performing and optimizing non-target data processing for $\mathrm{GC} \times \mathrm{GC}-\mathrm{MS}$. A set of 29 mouse liver samples was analyzed with both GCMS and GC $\times$ GC-MS. The same set of samples were analyzed with both systems, both systems used identical injectors, injection methods and gas chromatographs. Therefore, the variability in the RSDs of internal standards and QC samples was caused by later stages of the analytical process (i.e. $\mathrm{GC}$ temperature ramp, second-dimension GC separation versus no second-dimension GC separation, detection and processing). Ideally, the same detectors should be used to compared one-dimensional and twodimensional processing. However, in our experience the Chemstation software allows a more precise quantification in one-dimensional processing than can be achieved with the ChromTOF software, e.g. due to (i) the possibility to set integration parameters for individual peaks, (ii) the ability to use qualifier masses (define ratios between masses that should be fulfilled to assign a target) and (iii) the absence of an automated deconvolution process. Therefore, we used an Agilent GC-quadrupole MS system for this comparative study (and the majority of metabolomics studies in our lab), even though the time-of-flight mass spectrometer is more sensitive.

The fully optimized GC-MS processing method had a targeted approach (see Sect. 2). All 170 quantified metabolites in all samples were automatically integrated, the integration results visually inspected and wrongly integrated peaks were manually corrected. The non-targeted GC $\times$ GC-MS data processing method was semiautomated, i.e. the construction of the target table and assignment of missing values required manual interaction; however, the integration of peaks or mistakes in the combination of peaks from the same entry were not corrected to reduce the processing time. The data-processing times and results for GC-MS and GC $\times$ GC-MS were compared. Furthermore, the general data quality of the $\mathrm{GC} \times \mathrm{GC}-\mathrm{MS}$ analyses was investigated. Finally, the results obtained with the liver samples using GC-MS and $\mathrm{GC} \times \mathrm{GC}-\mathrm{MS}$ were analyzed using multivariate statistics (PCA/PCDA) in order to identify time-resolved metabolite patterns. These data may provide biomarkers for the development and progression of insulin resistance/DM2 and insight into the metabolic dysregulations underlying the disease process. 
3.1 Comparison of time required for processing of $\mathrm{GC}-\mathrm{MS}$ and $\mathrm{GC} \times \mathrm{GC}-\mathrm{MS}$ data

The workflow for the GC $\times$ GC-MS processing is shown in Table 1 . In total approximately $50 \mathrm{~h}$ of analyst time were required to optimize the processing and subsequently process the entire data set of 29 samples. Furthermore approximately $61 \mathrm{~h}$ of computer time were needed for the processing. The processing of the data files with the computer was mostly done overnight, so that the optimization and processing of the data set could be performed within about 2 weeks. The GC-MS processing required about $40 \mathrm{~h}$ of analyst time; $25 \mathrm{~h}$ for the construction of the target table (including finding the targets by using the in-house database of commonly found metabolites, searching new targets in this specific study and adjusting integration parameters for individual metabolites) and $15 \mathrm{~h}$ for the processing of one batch and eventual correction of the integration of metabolites from the calibration table. Both processing methods are quite time consuming, especially for the GC $\times$ GC-MS data, requiring $25 \%$ more analyst time compared to GC-MS processing. However, the optimization and construction of the target table takes a relatively long time, and every extra batch of about 30 samples will take approximately 20 and $10 \mathrm{~h}$ of analyst time for, respectively, 2D and 1D-processing. For the $\mathrm{GC} \times \mathrm{GC}-\mathrm{MS}$ processing, the assignment of the missing values is very time consuming (Table 1, step 8), even though we used a strategy to fill the missing values by reprocessing the data with a very low match threshold of 200 in the calibration table (cf. Sect. 2.12). This strategy decreased the required analyst time for this step (Table 1, step 8) with approximately $30-50 \%$ from $30 \mathrm{~h}$ to about 15-20 $\mathrm{h}$ per batch. However, one major bottleneck in the processing was the speed of the software in combination with the computer speed. Due to the large data files obtained with GC $\times$ GC-MS every action performed in the software is very slow, e.g. loading of a data file, selection of an assigned peak in the calibration table and the assignment of a peak to an entry in the calibration table and especially the correction of wrongly integrated and combined modulations from one entry. So even if no manual corrections are performed, the checking of the data is already very time-consuming. This might be improved using a faster computer, however, it is expected that the gain in speed will be limited. Probably, more efficient algorithms are required to improve the speed significantly. It should be noted that recently a newer and faster version of the ChromaTOF software (V 4.30) has become available. Most probably the computer processing time and the time required for the assignment of the blank values (step 8 , Table 1) can be reduced with the new software.

3.2 Comparing data processing results of onedimensional GC-MS with two-dimensional $\mathrm{GC} \times \mathrm{GC}-\mathrm{MS}$

\subsubsection{Number of entries}

The target table for GC-MS was constructed using a homemade database with retention times and mass spectra of over 600 reference metabolites (authentic standards), over 100 annotated metabolites (spectral match with NIST library) and over 200 unknown metabolites commonly found in blood products. Additionally, compounds detected specifically for this study in the QC sample (see Sect. 2) were added to the target table. A total of 170 targets were found in the liver samples, some with very low signal-tonoise ratios $(\mathrm{S} / \mathrm{N}=3$ in reconstructed ion chromatogram (RIC)). In Table 2 the number of entries above a certain signal-to-noise ratio in the total ion current (TIC) after

Table 1 Workflow for optimizing and carrying out GC $\times$ GC-MS data processing

\begin{tabular}{|c|c|c|c|}
\hline Task & Samples & $\begin{array}{l}\text { Analyst } \\
\text { time (h) }\end{array}$ & $\begin{array}{l}\text { Computer } \\
\text { time }(\mathrm{h})\end{array}$ \\
\hline $\begin{array}{l}\text { 1. Optimize processing method (peak width, smoothing, match required } \\
\text { to combine 2D peaks from one entry) }\end{array}$ & $5 \mathrm{QCs}$ & 8 & 20 \\
\hline 2. Processing for construction of target table & $1 \mathrm{QC}$ & 0 & 1 \\
\hline $\begin{array}{l}\text { 3. Construction target table (removing artifacts from, i.e. } \\
\text { solvent and reagents) }\end{array}$ & $1 \mathrm{QC}$ & 1 & 0 \\
\hline 4. Find targets from $\mathrm{GC}-\mathrm{MS}$ in $\mathrm{GC} \times \mathrm{GC}-\mathrm{MS}$ target table & $1 \mathrm{QC}$ & 16 & 0 \\
\hline 5. Processing of samples using constructed target table & 29 (all) & 0 & 40 \\
\hline 6. Copy data to spreadsheet & 29 (all) & 1 & 0 \\
\hline 7. Removing entries with too many blanks & 29 (all) & 1 & 0 \\
\hline 8. Assigning peaks of remaining blank values ${ }^{a}$ & 29 (all) & 20 & 0 \\
\hline Total time required & 29 (all) & 47 & 61 \\
\hline
\end{tabular}

${ }^{a}$ Reprocessing of all the data with a low match factor (200) takes about $20 \mathrm{~h}$ of extra computer time 
Table 2 Number of entries in a GC-MS and GC $\times$ GC-MS chromatogram of a pooled mouse liver sample

\begin{tabular}{lcc}
\hline S/N ratio $^{\text {a }}$ & GC-MS & GC $\times$ GC-MS \\
\hline S/N $>3$ & 435 & 3770 \\
S/N $>50$ & 165 & 1905 \\
S/N $>100$ & 96 & 1223 \\
S/N $>200$ & 52 & 835 \\
S/N $>500$ & 11 & 518 \\
\hline
\end{tabular}

${ }^{a}$ Signal-to-noise ratio in the total ion current

b Number of entries obtained by deconvolution software (see text for details) and after removal of artifacts from, i.e. solvents and reagents (eluting at low ${ }^{2} t_{R}$ )

deconvolution are shown for GC-MS (determined with AMDIS deconvolution software; Stein 1999) and $\mathrm{GC} \times \mathrm{GC}-\mathrm{MS}$. Due to lower detection limits and higher peak capacities in GC $\times$ GC-MS compared with GC-MS, more possible compounds were detected.

For the construction of the GC $\times$ GC-MS target table an $\mathrm{S} / \mathrm{N}$ cutoff of 200 was chosen. It should be mentioned that this $\mathrm{S} / \mathrm{N}$ is calculated for the unique mass (RIC) determined by the software, rather than the $\mathrm{S} / \mathrm{N}$ ratio in the total ion current used in Table 2. Therefore, the number of entries at this cut-off value were higher than in Table 2, i.e. 1034 entries were found with a $\mathrm{S} / \mathrm{N}>200$. It should be mentioned that for some metabolites due to the oximation two peaks can be obtained for one metabolite, so the actual number of metabolites detected is lower than the number of entries found.

\subsubsection{RSDs of internal standards}

The quantification of the internal standards was checked and errors in the integrations were corrected manually for both methods. The RSDs of the MS response for the labelled internal standards were calculated for both the GC-MS and the GC $\times$ GC-MS methods (Table 3). The

Table 3 Comparing the RSDs of normalized MS response ${ }^{\mathrm{a}}$ for the internal standards for GC-MS and GC $\times$ GC-MS in all samples $(\mathrm{QC}$ and study samples)

\begin{tabular}{lll}
\hline Compound & \multicolumn{2}{l}{ RSD of MS response $(\%)$} \\
\cline { 2 - 3 } & GC-MS & GC $\times$ GC-MS \\
\hline Alanine-d4 & 7 & 8 \\
Leucine-d3 & 9 & 8 \\
Glutamic acid-d3 & 17 & 8 \\
Phenylalanine-d5 & 13 & 7 \\
Cholic acid-d4 & 4 & 6 \\
\hline
\end{tabular}

${ }^{\text {a }}$ MS responses of the internal standards were corrected for variations in injection volume and MS response by normalization on the response of dicyclohexylphthalate (used as injection standard) chromatographic performance of the $\mathrm{GC} \times \mathrm{GC}-\mathrm{MS}$ method was comparable or even better than that of the GCMS method, i.e. the RSDs of phenylalanine-d5 and glutamic acid-d3 were significantly better in the $\mathrm{GC} \times \mathrm{GC}-$ MS data compared to the GC-MS data and the other RSDs were comparable in both datasets. These results were in agreement with the results in Koek et al. (2008); in this study better RSDs were obtained compared to GC-MS for a representative set of metabolites, in addition better peak shapes and an improved mass loadability were obtained.

\subsubsection{RSDs in pooled QC samples}

A set of pooled mouse-liver samples were used as QC samples. These samples were injected at the beginning and at the end of the sequence and between every six samples. In total five QC samples were measured over the course of the study. The RSDs of the MS response of target compounds that were found with both $1 \mathrm{D}$ and $2 \mathrm{D} \mathrm{GC}-(\times \mathrm{GC})-$ MS (total 107 targets) were compared. The RSDs for all compared metabolites are shown in Table S1 in the supplement.

For the majority of metabolites (70 metabolites) similar RSDs were found with both methods $(<10 \%$ difference in RSDs), although generally the values of the RSDs for the GC $\times$ GC-MS data were slightly higher than obtained with the semi-automated GC-MS processing (Fig. 1). However, it should be taken into account that the comparison is somewhat biased towards the GC-MS method. Because the targets from the GC-MS list were searched for in the GC $\times$ GC-MS data and not the other way around, metabolites that performed well in the $\mathrm{GC} \times \mathrm{GC}-\mathrm{MS}$ method and were not present in the GC-MS target list or

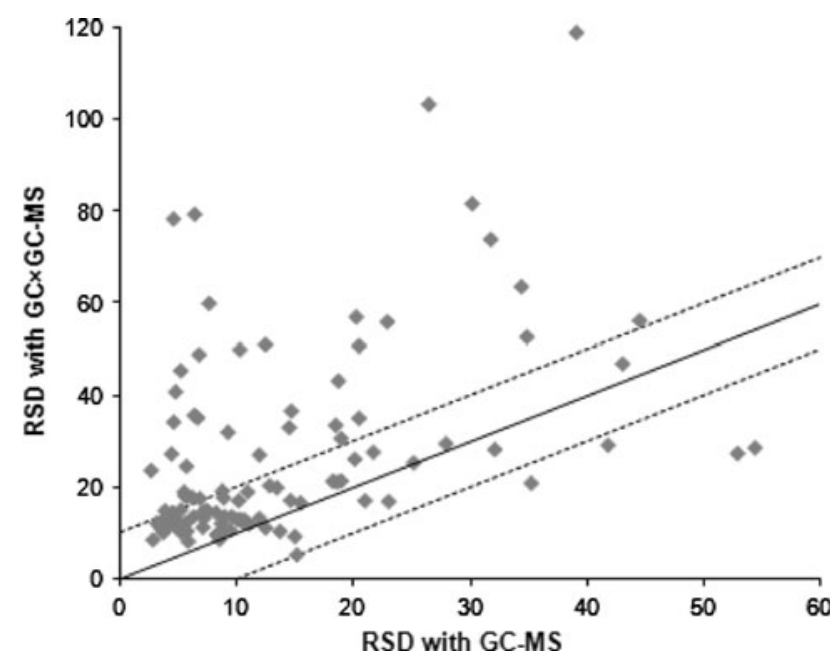

Fig. 1 Comparison of RSDs for metabolites in the QC samples of GC-MS and GC $\times$ GC-MS data after processing; only metabolites detected in both methods are shown 
were not detected with the GC-MS method (due to higher detection limits) were not evaluated in this comparision.

For six metabolites, better RSDs (differences in RSD more than $10 \%$ ) were obtained with the $\mathrm{GC} \times \mathrm{GC}-\mathrm{MS}$ method. For 37 metabolites poorer RSDs (differences in RSD more than $10 \%$ ) were obtained in the GC $\times$ GC-MS processing. The chromatographic performance of $\mathrm{GC} \times$ GC-MS was comparable when compared visually, or even better than the performance of GC-MS; this is in agreement with the comparable or even better RSD's for the manual corrected integration results of the internal standards (see above); the poorer RSD values for the notmanually corrected peaks with $\mathrm{GC} \times \mathrm{GC}-\mathrm{MS}$ were therefore caused by errors in the data processing. Seven of these compounds were overloaded (S-Table 1), which resulted in split peaks in the second dimension. Obviously these peaks will not be integrated correctly in an automated fashion, neither in GC-MS nor GC $\times$ GC-MS. In the GC-MS processing method overloaded peaks were manually integrated and therefore better RSDs were obtained.

For most other peaks the higher RSDs resulted from errors in the combining of ${ }^{2} \mathrm{D}$ peaks belonging to the same metabolite. For peaks to be combined the match between the mass spectra of different modulation cycles should meet the required match factor as set in the software. Decreasing the match required to combine, however, would risk combining peaks that originate from different metabolites, especially because masses $73\left(\left(\mathrm{CH}_{3}\right)_{3} \mathrm{SiOH}\right)$ and $147\left(\left(\mathrm{CH}_{3}\right)_{3} \mathrm{SiOSi}(\mathrm{CH} 3)_{2}\right)$ are dominant masses in the mass spectra of silylated compounds. Furthermore, in most cases the problems with combining peaks was due to deconvolution faults, and decreasing the match factor would not be an option in these cases. For nine compounds isomeric interferences of a close eluting peak was the cause of the combination error (S-Table 1). Due to the nature of the derivatization, two distinct compounds are formed for, for example, sugars and sugar-phosphates (cis- and transoxime forms). These two forms of one sugar elute close together in the first dimension and posses identical mass spectra. In these cases the chance of wrong assignment of the identity or mistakes in the combination of second dimension peaks is high. Another obstacle that impaired the quantification of seven metabolites was the assignment of the unique mass in the mass spectrum by the ChromaTOF software. For these compounds the non-selective masses m/z 73 or m/z 147 were assigned as unique masses, while these masses are present in all mass spectra of silylized compounds. Due to interferences of (partly) coeluting compounds the integration of these metabolites was inaccurate. In principle, the masses used for quantification of these metabolites can be manually adjusted in the calibration table, and these mistakes can probably be avoided by selecting a more selective mass instead of $\mathrm{m} / \mathrm{z} 73$ or $\mathrm{m} / \mathrm{z}$ 147. However, this requires extra time to check all automatically chosen quantification masses in the calibration table.

In summary, 70 metabolites were quantified correctly and 37 metabolites were quantified less accurately with the semi-automated $\mathrm{GC} \times \mathrm{GC}-\mathrm{MS}$ data processing method compared to the semi-automated GC-MS processing. For seven of the less-accurately quantified peaks the cause for the less good quantification could not be attributed to the ChromaTOF software, but to overloading effects. Thus, the semi-automated GC $\times$ GC-MS data processing method worked for $70 \%$ of the evaluated metabolites as good as the manually corrected GC-MS reference method.

\subsubsection{Summary on $G C \times G C-M S$ data quality}

The goal in this study was to assess the feasibility of using a processing strategy based on commercially available software (i.e. ChromaTOF software, LECO) for the unbiased, non-target semi-automated quantification of as many metabolites as possible in mouse liver samples measured with GC $\times$ GC-MS. The RSDs of the MS response for all entries were calculated in the QC samples. In Fig. 2 an overview of the amount of entries per RSD-range is given for both GC-MS and GC $\times$ GC-MS. In the GC $\times$ GC-MS
Fig. 2 Overview of the number of entries in the QC samples per RSD-range for GC-MS and $\mathrm{GC} \times \mathrm{GC}-\mathrm{MS}$

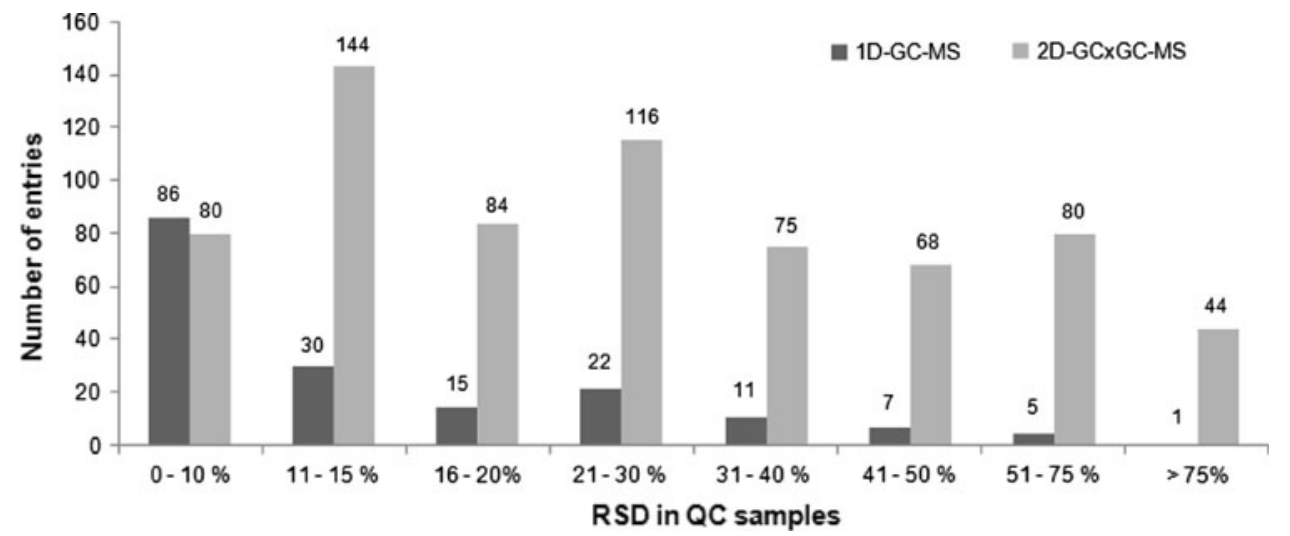


data, using a non-targeted approach, still 224 entries fit the strict FDA requirements for targeted analysis in bioanalysis (RSD <15\%) (U.S. Department of Health and Human Services et al. 2001), compared to 116 entries from the GC-MS data. This illustrates the added value of GC $\times$ GC-MS compared to GC-MS. In addition, the entries with higher RSDs still contain useful information, when the differences between the compared groups of samples are larger than the analytical variation, as illustrated below (cf. Sect. 3.3).

\subsection{Application}

The aim of the $\mathrm{GC}(\times \mathrm{GC})-\mathrm{MS}$ study was to investigate the influence of a high fat diet on the metabolite profiles in the liver. A total of 24 mouse liver samples, i.e. $t=0$ weeks $(n=8), t=6$ weeks $(n=8)$ and $t=12$ weeks $(n=8)$ after the start of the high fat diet, were measured with GC $\times$ GC-MS and GC-MS. Development of insulin resistance was monitored in individual mice over time by performing glucose tolerance tests and measuring specific biomarkers in plasma, and hyperinsulinemic-euglycemic clamp analysis to assess insulin resistance in a tissue-specific manner as described by Kleemann et al. (2010). After 6 weeks the mice had developed insulin resistance in the liver and after 12 weeks also in skeletal muscle and fat tissue (white adipose tissue).

With $\mathrm{GC} \times \mathrm{GC}-\mathrm{MS}$ many more compounds were measured than with GC-MS. Typical chromatograms of a mouse liver sample measured with GC-MS and GC $\times$ GC-MS are shown in Figs. 3 and 4. A total of 170 and 691 metabolites were quantified with GC-MS and GC $\times$ GC-MS, respectively, and analyzed using PCA and PCDA. First, the peaks that were present in the GC-MS as well as in the GC $\times$ GC-MS data set, hereafter referred to as overlap data, were analyzed with PCA (Fig. 5) and PCDA (Figure S-1, cf. Supplement). The first principal component (PC1) in PCA and the first linear discriminant

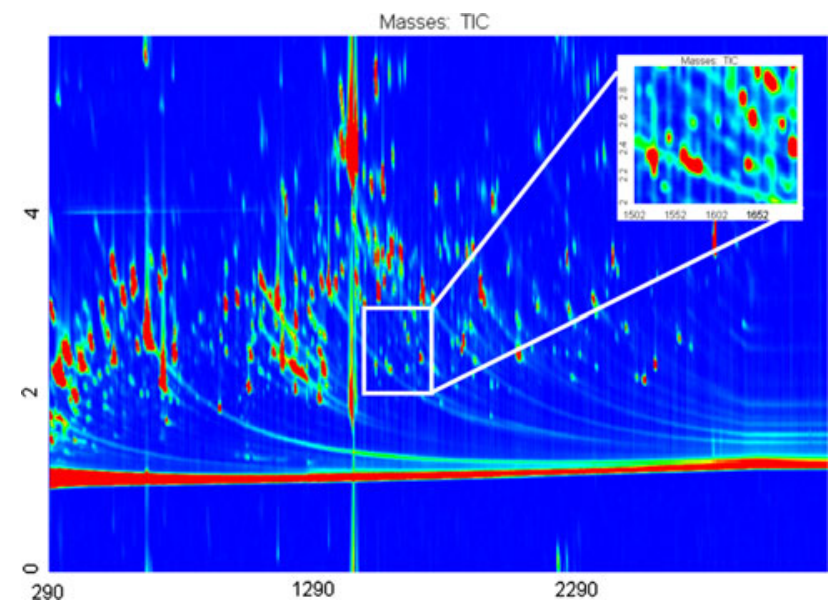

Fig. 4 Two-dimensional colour plot of a total ion GC $\times$ GC-MS chromatogram of a pooled mouse liver sample

(LD1) in PCDA mostly explained the variance between the $t=0$ samples and the $t=6$ and $t=12$ samples, PC2 (PCA) and LD2 (PCDA) explained some of the variance between $t=0$ and $t=12$ compared to $t=6$ weeks. As can be seen from Fig. 5 the results from both overlapping data sets were very comparable and the groups of mice from the different time points were rather well separated. The mahalanobis distances, i.e. a measure for the separation of the groups taking into account the spreading of the samples within one group, were calculated in the overlap data after PCA analysis (Mahalanobis 1930) (Table 4); the distances between the $t=0$ (baseline) and the high-fat-diet treated groups in the GC $\times$ GC-MS data was clearly improved, only the separation of the $t=6$ and $t=12$ groups was slightly better in de GC-MS data set. Furthermore, more than ten metabolites that were found in the top 20s of metabolites with the highest loadings in the GCMS and GC $\times$ GC-MS data in PCDA (LD1 and LD2, data not shown), were the same for both data sets indicating the similarity of the two overlapping data sets. Consequently, although the RSDs of responses for the metabolites in the
Fig. 3 Total-ion GC-MS chromatogram of a pooled mouse-liver sample

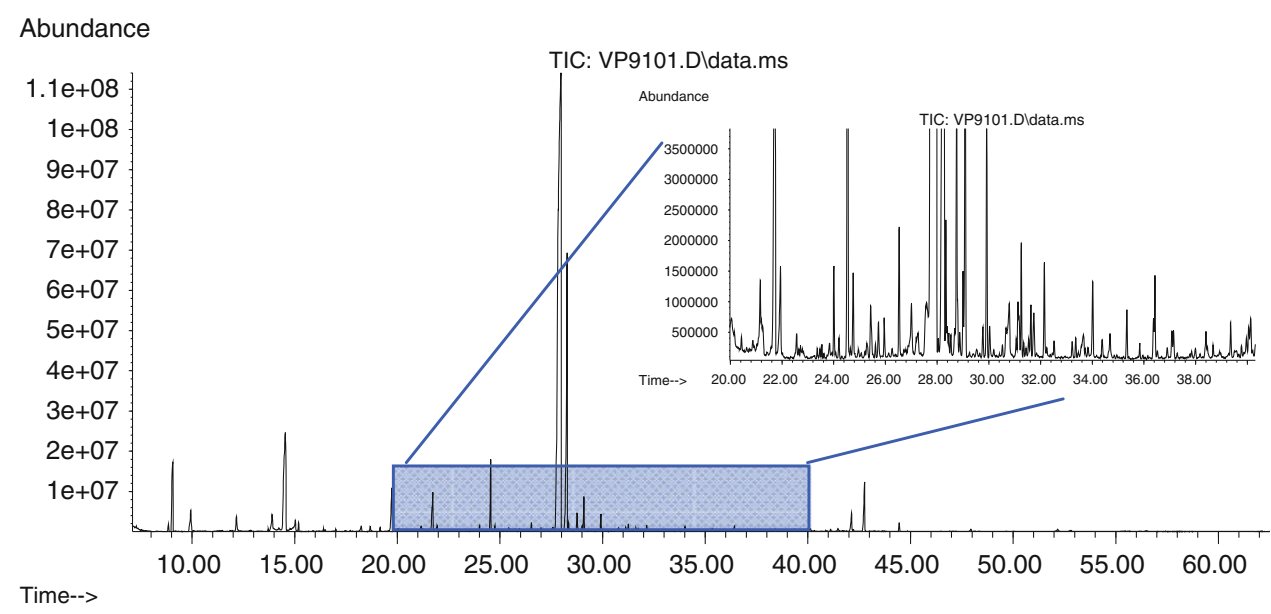


Fig. 5 PCA analysis of the overlap data (107 entries) for GC-MS (a) and GC $\times$ GC-MS (b) of mouse liver after 0 (red, + ), 6 (blue, $\Delta$ ) and 12 (green, •) weeks (Color figure online)

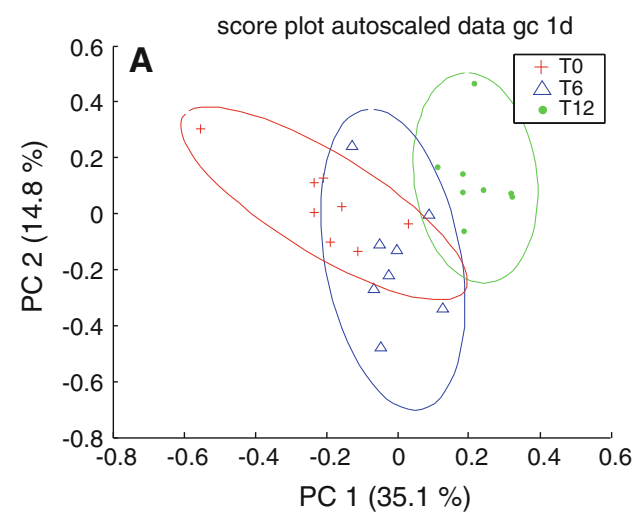

Table 4 Mahalanobis distances in the overlap data after PCA analysis of GC-MS and GC $\times$ GC-MS

\begin{tabular}{lll}
\hline Groups & \multicolumn{2}{l}{ Mahalanobis distance between groups } \\
\cline { 2 - 3 } & GC-MS & GC $\times$ GC-MS \\
\hline$t=0$ and $t=6$ weeks & 36 & 47 \\
$t=0$ and $t=12$ weeks & 44 & 74 \\
$t=6$ and $t=12$ weeks & 13 & 10 \\
\hline
\end{tabular}

$\mathrm{GC} \times \mathrm{GC}-\mathrm{MS}$ data were somewhat higher than in the GC-MS data, the biological information was preserved and even a slightly better group separation was obtained.

In Fig. 6 the result of the PCA analysis of the additional entries from the $\mathrm{GC} \times \mathrm{GC}-\mathrm{MS}$ data set (all entries minus overlap entries) compared to the GC-MS data set is shown (PCDA is shown in supplement Figure S-2). Again, the groups of mice from different time points were rather well separated, indicating the biological relevancy of the additional peaks in explaining the differences between the three

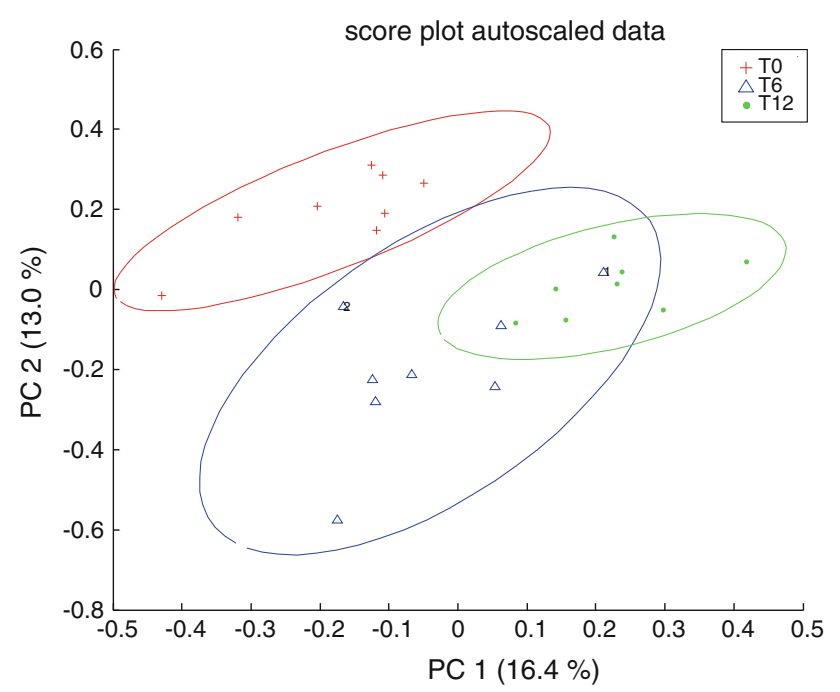

Fig. 6 PCA analysis of additional entries (compared to GC-MS; 584 peaks) in GC $\times$ GC-MS of mouse liver after $0($ red,+$), 6($ blue,$\Delta)$ and 12 (green, $\bullet$ ) weeks (Color figure online) groups, i.e. these additional metabolites contain information on the differences in metabolic profiles during the development of insulin resistance. It should be mentioned that 170 entries were detected in GC-MS and only 107 of these metabolites were assigned in the GC $\times$ GC-MS data set. The remaining 63 entries in GC-MS, except for a maximum of six high boiling compounds ( $\mathrm{Mw}_{\text {underivatized }}$ $>600$ ), are probably also present in the GC $\times$ GC-MS data set. However, these metabolites could not be unambiguously assigned, as no reference standards were available and/or their mass spectrum were not unique. As the first dimension column in $\mathrm{GC} \times \mathrm{GC}$ was a polar BPX50 column rather than an apolar DB5 column used for the GC-MS, the elution order and retention times differed for the first dimension separation, and retention times could not be used to identify metabolites found with one method in the other method.

The added value of GC $\times$ GC-MS is also demonstrated when the top 20 metabolites with the highest loadings in PCDA (LD1) of the total GC-MS and the total GC $\times$ GCMS data set are compared (Table 5). Most of the metabolites with high loadings in LD1 of GC-MS data are found in the top $20 \mathrm{LD} 1$ of GC $\times$ GC-MS (bold in Table 5) or are slightly lower ranked with still good loadings (italics in Table 5). With GC $\times$ GC-MS, in LD1 of the PCDA model many additional metabolites with high loadings were found. Only in a few cases the metabolites of the top 20 of GC-MS ranked lower due to poorer repeatability (i.e. 1-palmitoyl-sn-glycero-3-phosphocholine and gluconic acid); actually, a few metabolites (e.g. taurine and tyrosine) ranked within the top 20 of GC $\times$ GC-MS and not in the top 20 of GC-MS due to better performance with $\mathrm{GC} \times \mathrm{GC}-\mathrm{MS}$. In summary, the $\mathrm{GC} \times \mathrm{GC}-\mathrm{MS}$ analysis provides many additional possible biomarkers for the development and progression of insulin resistance.

In Fig. 7 the box plots of the relative response of some high-ranked metabolites from the PCDA analysis of the GC $\times$ GC-MS data are shown; these metabolites could provide more insight in the metabolic processes involved in the development and progression of insulin resistance 
Table 5 Top 20 metabolites with highest loading in LD1 in PCDA analysis ${ }^{\mathrm{a}}$

\begin{tabular}{|c|c|c|c|c|}
\hline \multirow[t]{2}{*}{ Rank PCDA } & \multicolumn{2}{|l|}{$\mathrm{GC} \times \mathrm{GC}-\mathrm{MS}$} & \multicolumn{2}{|l|}{ GC-MS } \\
\hline & Compound $^{\mathrm{b}}$ & Loading LD1 & Compound & Loading LD1 \\
\hline 1 & Campesterol* & -4.41 & Campesterol* & -4.55 \\
\hline 2 & M0617 (unsaturated fatty acid methyl ester) & -4.36 & GA0502 & -4.37 \\
\hline 3 & M0480 & -4.29 & 1,2-Diglyceride ${ }^{c}$ & 4.28 \\
\hline 4 & M0071 & -4.27 & 1-Palmitoyl-L-alpha-lysophosphatidic acid & -4.22 \\
\hline 5 & Linoleic acid* & -4.17 & 1-Palmitoyl-sn-glycero-3-phosphocholine & -4.18 \\
\hline 6 & M0535 (purine) & -4.04 & Arachidonic acid* & -4.03 \\
\hline 7 & Taurine* & 4.00 & Linoleic acid* & -4.00 \\
\hline 8 & Tyrosine* & -3.99 & GA0123 & -3.91 \\
\hline 9 & M0221 (amino-organic acid) & -3.98 & Beta-Alanine & -3.83 \\
\hline 10 & M0444 & -3.92 & GA0514 & -3.82 \\
\hline 11 & M0651 & -3.91 & GA0510 & -3.81 \\
\hline 12 & M0593 (monoglyceride) & -3.90 & C20:1 fatty acid & 3.80 \\
\hline 13 & Spermidine* & -3.85 & Pipecolinic acid & -3.78 \\
\hline 14 & M0182 (piperidine) & -3.84 & Fumaric acid & -3.77 \\
\hline 15 & M0550 & -3.84 & 1-Monolinoleoylglycerol & -3.77 \\
\hline 16 & M0283 (pyrrolidinone) & -3.81 & 1-Monooleoylglycerol* & 3.76 \\
\hline 17 & 1,5-Anhydro-D-glucitol* & -3.80 & Gluconic acid* & -3.75 \\
\hline 18 & M0600 (polyunsaturated fatty acid) & -3.79 & GA0520 & -3.71 \\
\hline 19 & M0597 & -3.75 & GA0507 & -3.71 \\
\hline 20 & M0307 (deoxyglucose or isomer) & -3.72 & Spermidine* & -3.70 \\
\hline
\end{tabular}

${ }^{a}$ In bold: metabolites present in both top 20s, in italics: metabolites present in both datasets, but not the top 20 of GC $\times$ GC-MS data. Metabolites marked with an asterisk are identified with authentic standards, other metabolites given a name are annotated via their mass spectra (more information in Sect. 2). The retention times and quantification masses of unknowns (M-coded and GA-coded metabolites) are listed in the Supplement (S-Table 2)

b Tentative annotation via MS library match or characterization of type of metabolite based on the mass spectrum are given in brackets

${ }^{c}$ Metabolites were not identified in the GC $\times$ GC-MS data set, most likely due to uncertainty in the assignment of the identity (no reference standard available and mass spectrum not unique enough). Only number 3 and 4 from GC-MS were not measured with GC $\times$ GC-MS, because their elution temperature was too high. Most probably all other metabolites are present in the 2D data set, but under a different name (M-code). However, none of the unknown top 20 metabolites from the GC-MS data set were present in the top 20 of the GC $\times$ GC-MS data (checked manually via mass spectra)

caused by a high fat diet. For example, the campesterol concentrations (Fig. 7, compound A) were significantly lowered after 6 and 12 weeks of high fat diet. Campesterol is a phytosterol, i.e. a steroid derived from plants, which is known for its cholesterol-lowering properties when used as a food additive (de Graaf et al. 2002). Moreover, a low campesterol/sistosterol ratio in serum and liver has been associated with severe liver damage and liver transplantation in primary bilairy cirrhosis (Nikkila et al. 1992a, b). In addition, the relative responses of metabolites $\mathrm{B}, \mathrm{C}$ (linoleic acid), D (Fig. 7) and arachidonic acid were significantly lower after 6 and 12 weeks of high fat diet; metabolites B and D were only present in low concentrations and probably therefore not detected with GC-MS. These metabolites were all poly-unsaturated fatty acids that are considered beneficial for health (Buckley and Howe 2009; Furuhjelm et al. 2009; Panza et al. 2009; Yaqoob
2009). Lowered levels of these metabolites are associated with an increased risk of inflammation and promotion of subacute chronic inflammation in metabolic disorders, which is again related to the development of obesity and insulin resistance (Cai et al. 2005; Verschuren et al. 2009). Furthermore, our results are consistent with the observed elevated levels of hepatic inflammation markers determined in plasma of the mice used herein as well as the observed increased activity of intrahepatic proinflammatory transcription factors such as NFkB in liver biopsies adjacent to the ones used for the present study (Kleemann et al. 2010).

Finally, the relative responses of tyrosine, spermidine (Fig. 7, compounds E and F) and beta-alanine were lower in the groups of mice sacrificed at $t=6$ weeks and $t=12$ weeks, while the level of taurine was significantly increased. The levels of amino acids are known to fluctuate during the development of insulin resistance (Huffman et al. 
Fig. 7 Box plots of the relative concentrations of the PCDA variables in LD1: Campesterol (a; $P<0.0001 ;$ ), M0617 (poly unsaturated fatty acid methyl ester; $P<0.0001)(\mathbf{b})$ and linoleic acid $(\mathrm{c} ; P<0.0001)$ M0600 (poly-unsaturated fatty acid) $(\mathbf{d} ; P=0.0002)$, tyrosine (e; $P<0.0001)$ and spermidine (f; $P=0.0015$ ). $P$-values calculated with one-way ANOVA with $\alpha=0.05$.

Analysis of differences between groups with Tukey post-hoc testing resulted in significant differences between groups $t=0$ vs. $t=6$ and $t=0$ vs. $t=12$ for all metabolites
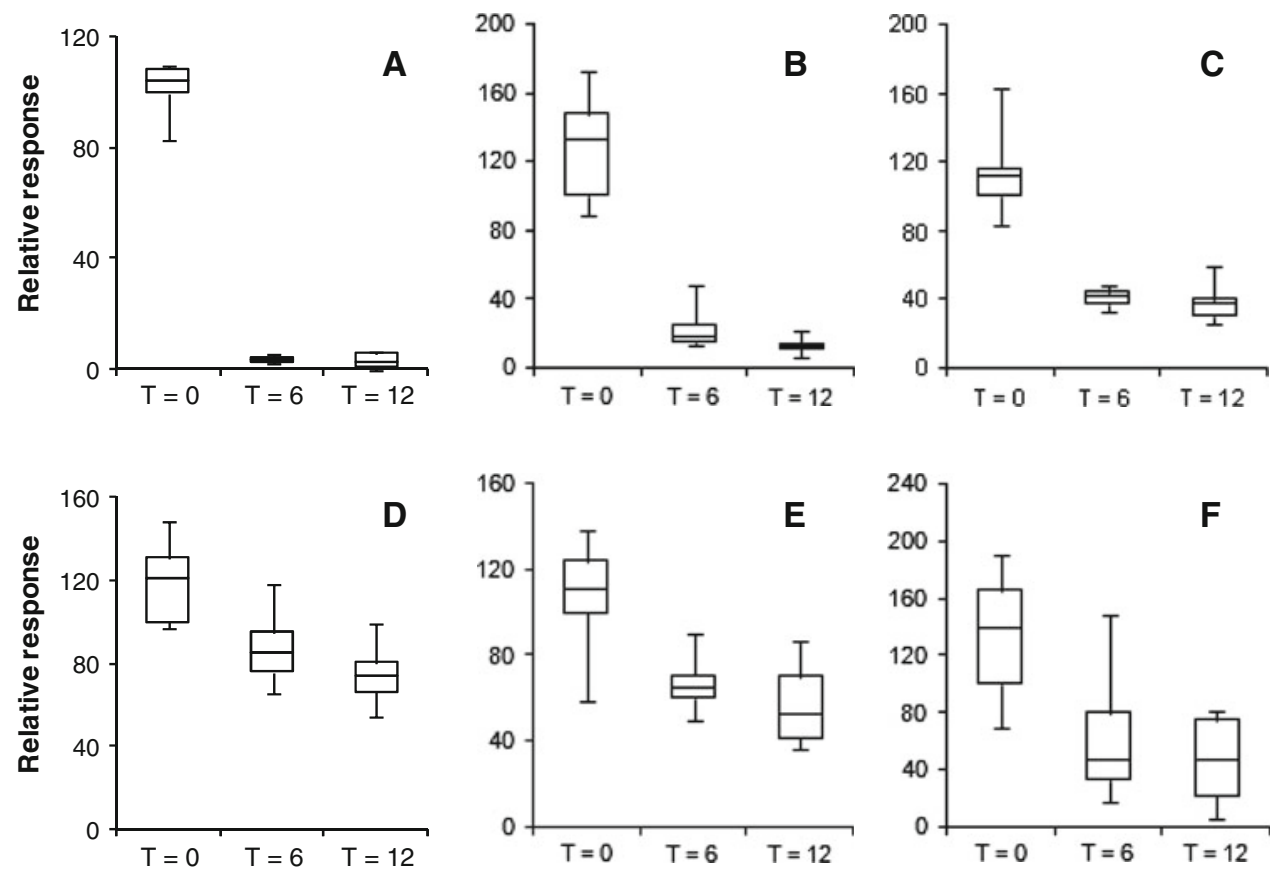

2009; Wijekoon et al. 2004). Interestingly, taurine was suggested to have beneficial effects by its ability to reduce intracellular oxidative stress generation and glycooxidation (Anuradha 2009), while this is the only metabolite in the GC $\times$ GC-MS PCDA top 20 that was significantly elevated in the animals after 6 and 12 weeks of high-fat diet. Furthermore, it is believed that certain amino acids play an important role in the development of diabetes and that dietary treatment with amino acids could prevent diabetes and diabetic complications (Anuradha 2009).

In conclusion, the added value of GC $\times$ GC-MS compared to GC-MS is clearly illustrated in this pre-clinical study. Although the RSDs of compounds in the QC samples for $\mathrm{GC} \times \mathrm{GC}-\mathrm{MS}$ were somewhat higher than in the GC-MS data, the biological information in the data was preserved. In addition, many more candidate biomarkers were detected that were significant in explaining the differences between the different sample groups in this study. Furthermore, the higher peak capacity resulted in cleaner mass spectra, facilitating the identification of possible biomarkers. Moreover, the position of the metabolite in the chromatogram (especially the ${ }^{2} \mathrm{r}_{\mathrm{T}}$ ) can also aid in the identification process (data not shown).

\subsection{Concluding remarks}

The feasibility of semi-automated non-target processing of GC $\times$ GC-MS data using commercially available software was assessed. A set of mouse liver samples was measured with GC-MS and GC $\times$ GC-MS and a total of 170 and 691 peaks, respectively, were quantified. The performance of the two methods was compared by evaluating the RSD values in the quality-control samples of metabolites present in both datasets. Although the chromatographic performance was comparable or even slightly better for GC $\times \mathrm{GC}-\mathrm{MS}$, as demonstrated for the manually integrated labelled internal standards, somewhat poorer RSDs for the relative responses of peaks determined in a semi-automated manner due to less accurate processing. Still a reliable and repeatable quantification was obtained for approximately $70 \%$ of the peaks, even though the integrations of the peaks from the GC $\times$ GC-MS data were not manually corrected in contrast with the GC-MS data.

In addition, $\mathrm{GC} \times \mathrm{GC}-\mathrm{MS}$ processing is time-consuming, the major bottleneck being the speed of the software tools and algorithms. However, application of the strategy described in this paper is feasible for small studies with a maximum of about 30-50 samples (eventually measured in duplicate). For the routine application of GC $\times \mathrm{GC}-\mathrm{MS}$ in metabolomics in larger studies, further improvement of data processing tools is required.

The mouse-liver samples were measured to study the development and progression of insulin resistance. The added value of GC $\times$ GC-MS was clearly illustrated, (i) over four times more peaks could be quantified, (ii) the biological information as acquired in GC-MS was preserved, (iii) several extra candidate biomarkers for the development of insulin resistance were found, and (iv) the superior peak capacity resulted in cleaner mass spectra, facilitating in principle the putative identification of metabolites.

Acknowledgement This study was supported by the research program of TNO Personalized Health 
Open Access This article is distributed under the terms of the Creative Commons Attribution Noncommercial License which permits any noncommercial use, distribution, and reproduction in any medium, provided the original author(s) and source are credited.

\section{References}

Anuradha, C. V. (2009). Aminoacid support in the prevention of diabetes and diabetic complications. Current Protein \& Peptide Science, 10(1), 8-17.

Buckley, J. D., \& Howe, P. R. (2009). Anti-obesity effects of longchain omega-3 polyunsaturated fatty acids. Obesity Reviews, $10(6), 648-659$.

Cai, D., Yuan, M., Frantz, D. F., Melendez, P. A., Hansen, L., Lee, J., et al. (2005). Local and systemic insulin resistance resulting from hepatic activation of IKK-beta and NF-kappaB. Nature Medicine, 11(2), 183-190.

de Graaf, J., De Sauvage Nolting, P. R., van Dam, M., Belsey, E. M., Kastelein, J. J., Haydn, P. P., et al. (2002). Consumption of tall oil-derived phytosterols in a chocolate matrix significantly decreases plasma total and low-density lipoprotein-cholesterol levels. British Journal of Nutrition, 88(5), 479-488.

Fiehn, O., Kopka, J., Trethewey, R. N., \& Willmitzer, L. (2000). Identification of uncommon plant metabolites based on calculation of elemental compositions using gas chromatography and quadrupole mass spectrometry. Analytical Chemistry, 72(15), 3573-3580.

Furuhjelm, C., Warstedt, K., Larsson, J., Fredriksson, M., Bottcher, M. F., Falth-Magnusson, K., et al. (2009). Fish oil supplementation in pregnancy and lactation may decrease the risk of infant allergy. Acta Paediatrica, 98(9), 1461-1467.

Hoggard, J. C., \& Synovec, R. E. (2008). Automated resolution of nontarget analyte signals in GC $\times$ GC-TOFMS data using parallel factor analysis. Analytical Chemistry, 80(17), 6677-6688.

Huffman, K. M., Shah, S. H., Stevens, R. D., Bain, J. R., Muehlbauer, M., Slentz, C. A., et al. (2009). Relationships between circulating metabolic intermediates and insulin action in overweight to obese, inactive men and women. Diabetes Care, 32(9), 1678-1683.

Jonsson, N., Gullberg, J., Nordstrom, A., Kusano, M., Kowalczyk, M., Sjostrom, M., et al. (2004). A strategy for identifying differences in large series of metabolomic samples analyzed by GC/MS. Analytical Chemistry, 76(6), 1738-1745.

Kleemann, R., van, E. M., Verschuren, L., van den Hoek, A. M., Koek, M., Wielinga, P. Y., et al. (2010). Time-resolved and tissue-specific systems analysis of the pathogenesis of insulin resistance. PLoS One, 5(1), e8817.

Kleemann, R., Verschuren, L., van Erk, M. J., Nikolsky, Y., Cnubben, N. H., Verheij, E. R., Smilde, A. K., Hendriks, H. F., Zadelaar, S., Smith, G. J., Kaznacheev, V., Nikolskaya, T., Melnikov, A., Hurt-Camejo, E., Van Der Greef, J., van, O.B., \& Kooistra, T. (2007) Atherosclerosis and liver inflammation induced by increased dietary cholesterol intake: A combined transcriptomics and metabolomics analysis. Genome Biology, 8(9), R200.

Koek, M. M., Muilwijk, B., van Stee, L. L. P., \& Hankemeier, T. (2008). Higher mass loadability in comprehensive two-dimensional gas chromatography-mass spectrometry for improved analytical performance in metabolomics analysis. Journal of Chromatography. A, 1186(1-2), 420-429.

Koek, M. M., Muilwijk, B., vander Werf, M. J., \& Hankemeier, T. (2006). Microbial metabolomics with gas chromatography/mass spectrometry. Analytical Chemistry, 78(4), 1272-1281.

Li, X., Xu, Z., Lu, X., Yang, X., Yin, P., Kong, H., et al. (2009). Comprehensive two-dimensional gas chromatography/time-of- flight mass spectrometry for metabonomics: Biomarker discovery for diabetes mellitus. Analytica Chimica Acta, 633(2), 257-262.

Mahalanobis, P. C. (1930). On tests and measures of group divergences. Journal of the Asiatic Society of Bengal, 26, 541-588.

Mohler, R. E., Tu, B. P., Dombek, K. M., Hoggard, J. C., Young, E. T., \& Synovec, R. E. (2008). Identification and evaluation of cycling yeast metabolites in two-dimensional comprehensive gas chromatography-time-of-flight-mass spectrometry data. Journal of Chromatography. A, 1186(1-2), 401-411.

Murphy, R. E., Schure, M. R., \& Foley, J. P. (1998). Effect of sampling rate on resolution in comprehensive two-dimensional liquid chromatography. Analytical Chemistry, 70(8), 1585-1594.

Nikkila, K., Hockerstedt, K., \& Miettinen, T. A. (1992a). High serum cholestanol and low campesterol/sitosterol ratio indicate severe liver damage and liver transplantation in primary biliary cirrhosis. Transplantation Proceedings, 24(1), 383-386.

Nikkila, K., Hockerstedt, K., \& Miettinen, T. A. (1992b). Serum and hepatic cholestanol, squalene and noncholesterol sterols in man: a study on liver transplantation. Hepatology, 15(5), 863-870.

O’Hagan, S., Dunn, W. B., Knowles, J. D., Broadhurst, D., Williams, R., Ashworth, J. J., et al. (2007). Closed-loop, multiobjective optimization of two-dimensional gas chromatography/mass spectrometry for serum metabolomics. Analytical Chemistry, 79(2), 464-476.

Oh, C., Huang, X., Regnier, F. E., Buck, C., \& Zhang, X. (2008). Comprehensive two-dimensional gas chromatography/time-offlight mass spectrometry peak sorting algorithm. Journal of Chromatography. A, 1179(2), 205-215.

Panza, F., Frisardi, V., Capurso, C., D’Introno, A., Colacicco, A. M., Di, P. A., et al. (2009). Polyunsaturated fatty acid and Sadenosylmethionine supplementation in predementia syndromes and Alzheimer's disease: A review. TheScientificWorldJournal, 9, 373-389.

Pierce, K. M., Hoggard, J. C., Hope, J. L., Rainey, P. M., Hoofnagle, A. N., Jack, R. M., et al. (2006a). Fisher ratio method applied to third-order separation data to identify significant chemical components of metabolite extracts. Analytical Chemistry, 78(14), 5068-5075.

Pierce, K. M., Hope, J. L., Hoggard, J. C., \& Synovec, R. E. (2006b). A principal component analysis based method to discover chemical differences in comprehensive two-dimensional gas chromatography with time-of-flight mass spectrometry $(\mathrm{GC} \times \mathrm{GC}-\mathrm{TOFMS})$ separations of metabolites in plant samples. Talanta, 70(4), 797-804.

Qiu, Y., Lu, X., Pang, T., Zhu, S., Kong, H., \& Xu, G. (2007). Study of traditional Chinese medicine volatile oils from different geographical origins by comprehensive two-dimensional gas chromatography-time-of-flight mass spectrometry (GC + GCTOFMS) in combination with multivariate analysis. Journal of Pharmaceutical and Biomedical Analysis, 43(5), 1721-1727.

Roessner, U., Wagner, C., Kopka, J., Trethewey, R. N., \& Willmitzer, L. (2000). Simultaneous analysis of metabolites in potato tuber by gas chromatography-mass spectrometry. Plant Journal, 23(1), 131-142.

Shellie, R. A., Fiehn, O., Welthagen, W., Zimmermann, R., Zrostlikovb, J., Spranger, J., et al. (2005). Statistical methods for comparing comprehensive two-dimensional gas chromatography-time-of-flight mass spectrometry results: Metabolomic analysis of mouse tissue extracts. Journal of Chromatography. A, 1086(1-2), 83-90.

Shellie, R., Marriott, P., \& Cornwell, C. (2001). Application of comprehensive two-dimensional gas chromatography $(\mathrm{GC} \times \mathrm{GC})$ to the enantioselective analysis of essential oils. Journal of Separation Science, 24, 823-830.

Sinha, A. E., Hope, J. L., Prazen, B. J., Nilsson, E. J., Jack, R. M., \& Synovec, R. E. (2004). Algorithm for locating analytes of interest based on mass spectral similarity in GC $\times$ GC-TOF-MS 
data: analysis of metabolites in human infant urine. Journal of Chromatography. A, 1058(1-2), 209-215.

Stein, S. E. (1999). An integrated method for spectrum extraction and compound identification from gas chromatography-mass spectrometry data. Journal of the American Society for Mass Spectrometry, 10(8), 770-781.

Strelkov, S., von Elstermann, M., \& Schomburg, D. (2004). Comprehensive analysis of metabolites in Corynebacterium glutamicum by gas chromatography/mass spectrometry. Biological Chemistry, 385(9), 853-861.

U.S. Department of Health and Human Services, Food and Drug Administration, Center for Drug Evaluation and Research (CDER), \& Center for Veterinary Medicine (CVM). (2001). Guidance for industry bioanalytical method validation. Rockville, MD: FDA/Center for Drug Evaluation and Research.

Verschuren, L., Kooistra, T., Bernhagen, J., Voshol, P.J., Ouwens, D. M., van, E. M., de Vries-van der Weij, J., Leng, L., van Bockel, J. H., van Dijk, K. W., Fingerle-Rowson, G., Bucala, R., \&
Kleemann, R. (2009). MIF deficiency reduces chronic inflammation in white adipose tissue and impairs the development of insulin resistance, glucose intolerance, and associated atherosclerotic disease. Circulation Research, 105(1), 99-107.

Villas-Boas, S. G., Hojer-Pedersen, J., Akesson, M., Smedsgaard, J., \& Nielsen, J. (2005). Global metabolite analysis of yeast: Evaluation of sample preparation methods. Yeast, 22(14), 1155-1169.

Wijekoon, E. P., Skinner, C., Brosnan, M. E., \& Brosnan, J. T. (2004). Amino acid metabolism in the Zucker diabetic fatty rat: effects of insulin resistance and of type 2 diabetes. Canadian Journal of Physiology and Pharmacology, 82(7), 506-514.

Yaqoob, P. (2009). The nutritional significance of lipids rafts. Annual Review of Nutrition, 29, 257-282.

Zadelaar, S., Kleemann, R., Verschuren, L., De Vries-Van Der Weij, J., Van Der Hoorn, J., Princen, H. M., \& Kooistra, T. (2007). Mouse models for atherosclerosis and pharmaceutical modifiers. Arteriosclerosis, Thrombosis, and Vascular Biology, 27(8), $1706-1721$ 